\title{
Visite virtuelle des salles blanches ESIEE
}

\author{
T. Grandpierre ${ }^{\mathrm{a}}$

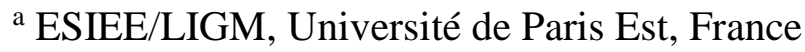 \\ Contact email : thierry.grandpierre@esiee.fr
}

\begin{abstract}
Nous présentons un système de réalité virtuelle qui a été mis en place afin de permettre à tous de visiter virtuellement les nouvelles salles blanches de l'ESIEE. C'est une visite immersive qui peut s'effectuer à l'aide d'un casque de réalité virtuelle ou bien dans une salle spécifique de projection que nous décrivons. Ce parcours virtuel permet de se déplacer dans toutes les pièces du bâtiment ainsi que de visiter les locaux techniques. Il offre le choix d'afficher des images de synthèse ou bien des photos panoramiques. Nous décrivons ici les outils utilisés ainsi que toutes les étapes qui ont mené à la réalisation de cette application.
\end{abstract}

\section{Introduction}

Les salles blanches, quelles qu'elles soient sont des équipements très souvent complexes, réservés à des usages très précis avec des conditions d'accès draconiennes pour ne pas les polluer. Elles font l'objet d'investissements souvent importants, que ce soit pour les construire ou pour maintenir leur état de propreté interne. Les équipements mis en place dans ces salles, sont eux souvent exceptionnels. Il existe cependant de nombreuses raisons de faire visiter ces salles, notamment pour la formation, la communication auprès du grand public, rendre compte aux financeurs...

Toutes ces visites sont peu compatibles avec le principe même de salles blanches où l'on souhaite minimiser les impuretés. Elles sont pourtant nécessaires. De plus chaque séjour en salle blanche prend du temps puisqu'il faut revêtir des tenues spécifiques (surchaussures, charlotte, combinaison, masque etc.) pour ne pas apporter d'impuretés. Parfois des couloirs de visites périphériques existent, ils permettent de visualiser l'intérieur des salles au travers de cloisons vitrées. Cependant, ces couloirs complexifient grandement l'architecture du bâtiment, ainsi que son utilisation en restreignant des zones de travail tout en alourdissant le coût de revient du bâtiment, tout cela sans permettre un accès aux manipulations qui s'y effectuent.

Nous proposons ici une solution qui a été mise en place à l'ESIEE pour répondre à ce besoin du public mais aussi pour la formation. Cette solution est basée sur une visite virtuelle immersive et interactive qui peut s'effectuer en utilisant différentes technologies de réalité virtuelle (RV), des casques immersifs à la plus complexe dans une salle de RV.

Cet article est organisé comme suit : la deuxième section présente les salles blanches de l'ESIEE, puis un panorama des technologies de RV avant d'exposer dans la troisième section la démonstration qui a été conçue à l'ESIEE pour les salles blanches de l'ESIEE. Enfin nous conclurons en proposant des perspectives à cette approche. 


\section{Les Salles Blanches ESIEE}

Après 30 ans de fonctionnement les anciennes salles blanches d'ESIEE Paris, école de la Chambre de Commerce et d'Industrie de Paris Ile-de-France, ont été mises à l'arrêt en octobre 2017 pour faire place à une nouvelle salle blanche. A l'issue de 18 mois de travaux qui avaient débuté en août 2015, un nouveau bâtiment regroupant la salle blanche ainsi que toutes les facilités a vu le jour (figure 1). Ce bâtiment de $2700 \mathrm{~m}^{2}$ répartis sur trois niveaux comprend $650 \mathrm{~m}^{2}$ de salle blanche de conception moderne avec une alternance de doigts gris et de zones de travail en blanc où les équipements sont positionnés en passe paroi (architecture en «peigne », figure 2). La gestion de l'air se fait via un plénum de soufflage muni de 80 FFU (Filter Fan Unit) pour le contrôle d'un flux laminaire et recyclé dans les puits de reprise à travers un faux plancher en aluminium perforé. La salle blanche inclut une zone de $58 \mathrm{~m}^{2}$ en classe 100 (ISO 5) dédiée à la lithographie et au scellement de substrats (figure 3) ; les autres zones sont en classe 10000 (ISO 7) à l'exception des doigts gris et de l'espace dédié à la découpe et au packaging qui sont en classe 100000 (ISO 8).

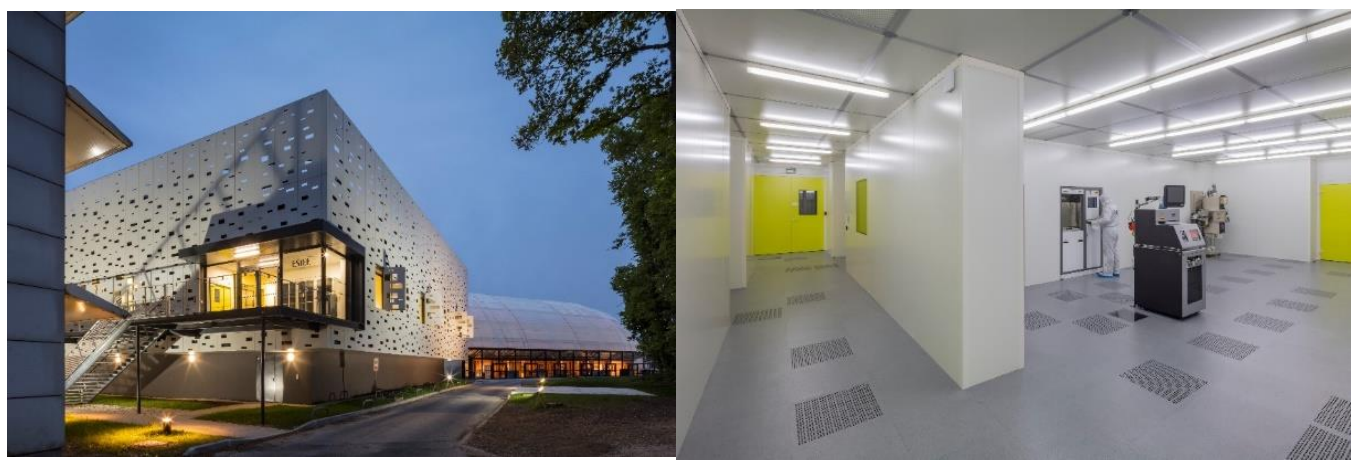

Fig.1. Photo du bâtiment extérieur (gauche), salle en classe 10000 / ISO 7 dépôts de couches minces (droite).

Située au cœur du campus de l'Université Paris-Est, ces salles Blanches sont destinées à des activités de formation en lien avec le Centre de Micro nanoélectronique de Paris Ilede-France (CEMIP) - pôle francilien du groupement d'intérêt public pour la Coordination Nationale pour la Formation en Micro-électronique et en nanotechnologies (CNFM). Elles visent aussi la recherche et l'appui aux entreprises autour des micro et nanotechnologies, en particulier dans les domaines des capteurs, des biocapteurs, de la micro-fluidique et de la récupération d'énergie.

\section{III.La réalité virtuelle}

\section{$\underline{\text { Définition }}$}

Aujourd'hui la RV est un terme très générique qui peut englober un très grand nombre de disciplines. Dans cet article la RV est définie comme le couplage de matériels et de logiciels qui permettent l'immersion d'une ou plusieurs personnes. La réalité est reproduite artificiellement afin de pouvoir y interagir. Selon Jaques Tisseau un système de RV doit permettre simultanément : l'immersion de l'utilisateur, son interaction avec le monde virtuel, et enfin le système doit être autonome (dans le sens vie en autonomie i.e. simulation). Les objectifs de la RV sont très variés [1] nous citerons le jeu, la formation, la culture, l'art. Et les domaines touchés sont innombrables : imagerie médicale, pharmacie, industries de l'Energie, construction, aéronautique, spatiale, automobile, géographie, etc. 


\section{Dispositifs matériels : Affichage}

Il existe trois grandes catégories de systèmes immersifs en RV. Les dispositifs peu immersifs basés sur un PC, une tablette, un smartphone avec lequel l'utilisateur peut interagir (par exemple se déplacer). Les dispositifs immersifs portables qui sont malheureusement limités à un utilisateur et isolent visuellement l'utilisateur du monde réel. Il s'agit des casques de RV (type HTC Vive, Occulus Rift, Gear VR), appelés HMD pour Head Mounted Device. Ils existent depuis longtemps mais la miniaturisation des écrans LCD haute résolution et l'accroissement de la puissance de calcul des ordinateurs a entrainé une forte démocratisation de cette technologie ces dernières années. En effet, le coût de ces systèmes les rend aujourd'hui abordables pour le grand public. Enfin, le troisième type de système correspond aux salles immersives basées sur des vidéoprojecteurs et plusieurs écrans de projection de grandes tailles (plusieurs mètres de diagonale) disposés en cube ou en courbe de façon à plonger un ou plusieurs utilisateurs dans la scène virtuelle. Ces systèmes sont souvent appelés CAVE (figure 2) qui signifie «Cave Automatic Virtual Environnent » (c'est en effet un acronyme récursif comme GNU ${ }^{1}$ ).

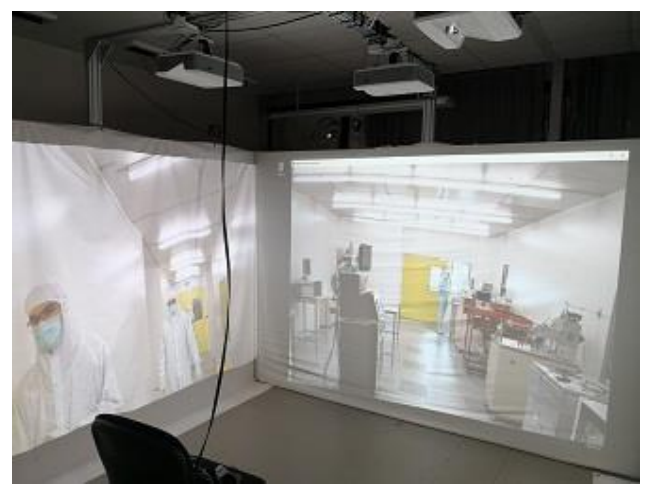

Fig.2. CAVE de l'ESIEE, on remarque les 3 vidéoprojecteurs au plafond.

\section{Dispositifs matériels : Capteurs}

Comme nous l'avons vu en introduction, il faut que le ou les utilisateurs puissent interagir avec la scène virtuelle, mais selon les systèmes il faut être capable de détecter l'orientation du regard de l'utilisateur. Pour l'interaction, il existe une grande diversité de capteurs dont le choix est étroitement lié au type d'immersion utilisé. Dans le cas des PC on utilisera généralement la souris ou une manette puisque facilement disponible. Dans le cas des tablettes c'est plutôt l'écran tactile et les capteurs d'orientation et d'inclinaison qui sont utilisés. Les casques immersifs sont souvent fournis avec des manettes spécifiques (i.e. Occulus Touch) permettant de localiser la position des manettes grâce à un couplage avec des caméras infrarouges (IR) qui permettent aussi de mesurer l'orientation de la tête. Pour cela, les manettes et le casque sont pourvus de leds IR. Le dispositif Leapmotion est aussi très souvent utilisé en RV sur PC et casque. Il repose sur un boitier équipé de 2 caméras IR placées sous les mains ou sur le casque afin de détecter la position des doigts et de permettre une interaction fine. Enfin, dans les CAVEs on utilise généralement la localisation grâce à des caméras IR, des projecteurs IR et des marqueurs réfléchissants collés (ou « scratchés ») sur les parties à détecter (main, bras, tête, etc.). Pour l'interaction fine on peut utiliser des gants de données (Datagloves) qui permettent de localiser la position, ou au moins la flexion des doigts sans restreindre l'espace de détection comme le fait la Leapmotion.

\footnotetext{
${ }^{1}$ Acronyme de GNU, célèbre fondation pour le logiciel libre : « GNU is Not Unix »
} 


\section{Dispositifs logiciel}

Quelle que soit la technologie d'affichage utilisée, il est nécessaire qu'un programme informatique calcule en temps réel les images afin de simuler un environnement réaliste et interactif. Ce programme utilise l'information des capteurs pour connaitre la position de l'utilisateur et recalculer l'image qu'il perçoit. La latence entre le mouvement de l'utilisateur et l'arrivée des photons sur la rétine de l'utilisateur ne doit pas dépasser 20ms sous peine de subir le «mal de la RV ». Il faut donc une forte puissance de calcul. Pour développer des applications de RV il existe aujourd'hui des environnements de développement intégrés qui permettent à la fois de modéliser les scènes virtuelles, les objets virtuels, de coder, compiler le programme et le déployer sur le matériel ciblé. Les leaders sont aujourd'hui Unity (gratuit mais non open source qui permet un usage commercial gratuitement jusqu'à une certaine limite, programmation en C\#), Unreal Engine 4 - UE4 (open source avec possibilité d'usage commercial gratuitement jusqu'à une certaine limite, programmation en $\mathrm{C}++$ ) et CryEngine (open source, gratuit, programmation en $\mathrm{C}++$ ). Avant ces environnements il était nécessaire de développer totalement l'application à l'aide de bibliothèques logicielles (OGRE, OpenSceneGraph OSG, etc.) ce qui requérait des compétences en programmation beaucoup plus fortes et diversifiées qu'actuellement.

\section{IV.La visite virtuelle}

A l'aide d'un groupe d'étudiants en projet de $3^{\text {ème }}$ année composé de Jules Caille, Lucas Dure et Guillaume Urard, nous avons développé une visite virtuelle des salles blanches de l'ESIEE. Cette visite est disponible à la fois pour casques immersifs et pour notre CAVE. C'est le logiciel UE4 (figure 3) qui a été choisi pour le développement de cette application pour deux raisons principales. D'une part, son code étant disponible (il est open source) il est possible de le modifier afin de pouvoir piloter notre CAVE et ses périphériques. D'autre part, il possède aujourd'hui un moteur de rendu graphique très évolué qui lui permet de générer en temps réel des images très réalistes, notamment dans la gestion des éclairages.

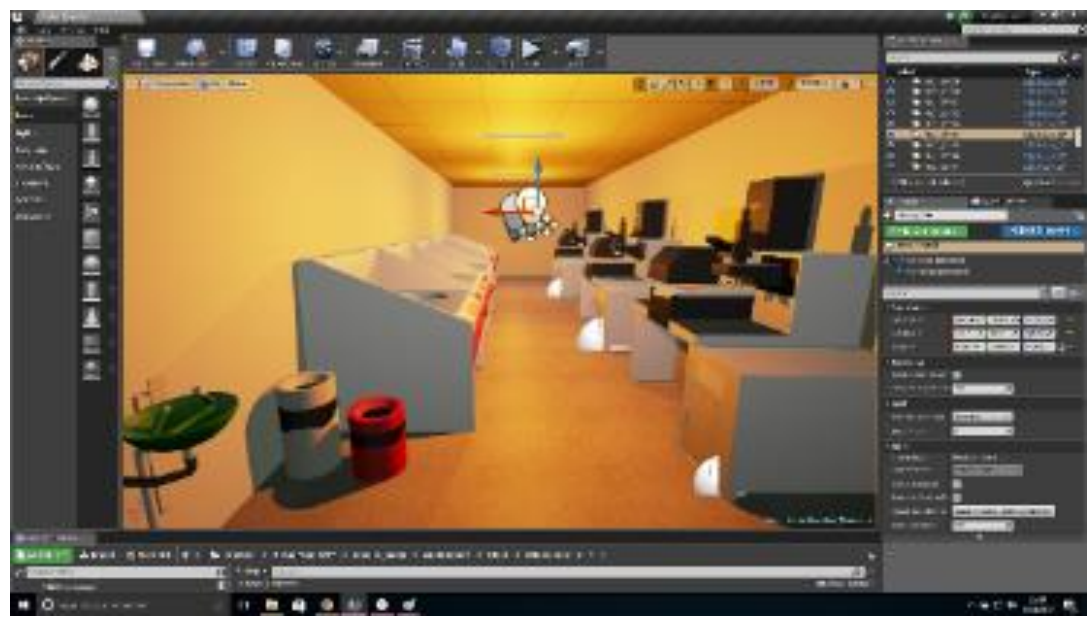

Fig.3. Modélisation d'une salle sous UE4.

La conception a débuté par une visite réelle des salles blanches, avec prise de mesures et de photographies à $360^{\circ}$ (figure 4) à l'aide de smartphones. Ces photos panoramiques sont obtenues à partir de prises de vues dans toutes les directions, à $360^{\circ}$. Chaque salle et 
équipements ont ainsi été soigneusement photographiés, y compris les installations techniques des salles blanches.

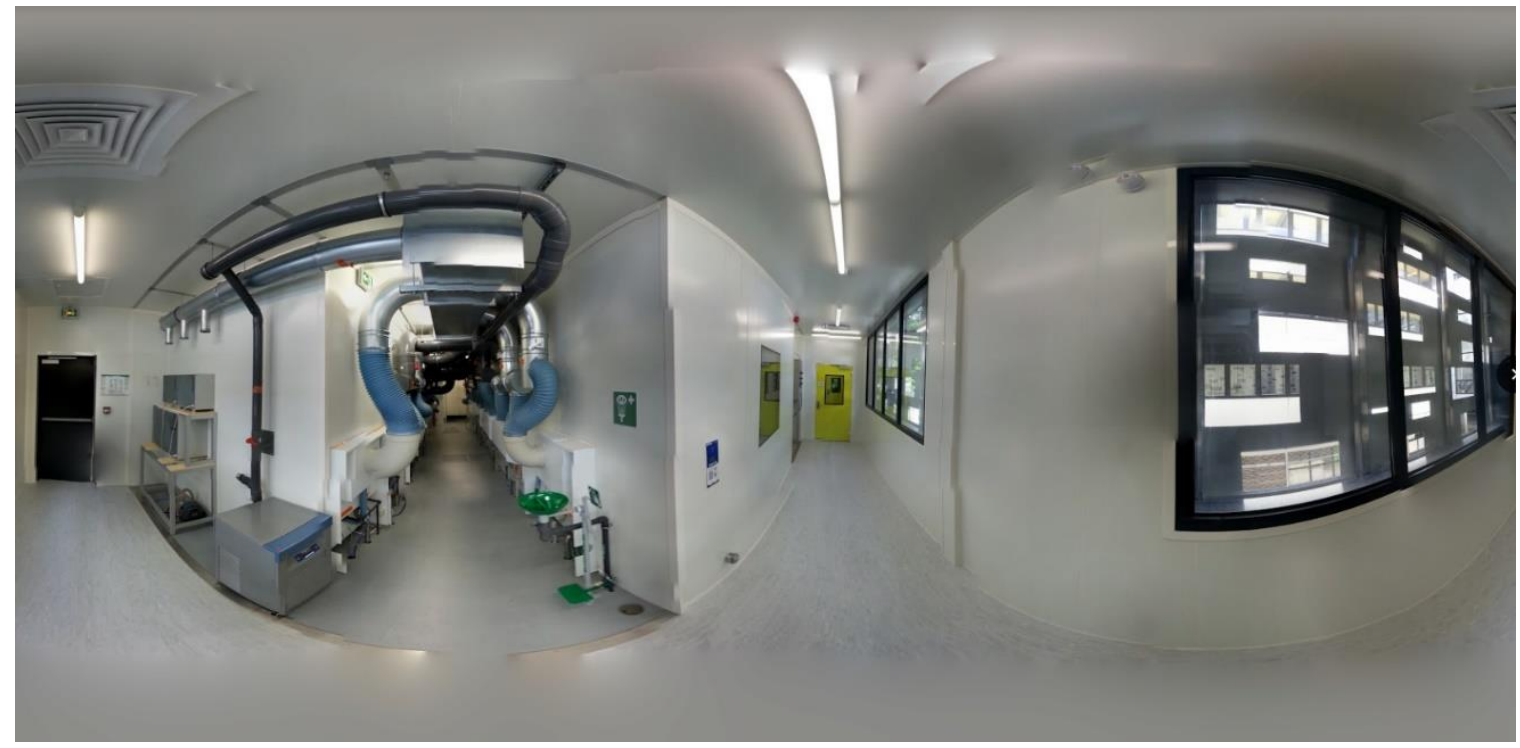

Fig.4. Photo à $360^{\circ}$ des locaux techniques : aspiration des hottes.

A partir de ces éléments et des plans de construction dont nous disposons, le bâtiment et une grande partie des équipements ont été modélisés sous Blender (logiciel de modélisation open source) mais aussi directement dans l'éditeur graphique UE4 (pour les murs, sols, toit et cloisons du bâtiment). Toutes les salles du premier étage ont été modélisées, un travail de fourmi dont les figures 5 et 6 présentes quelques extraits.

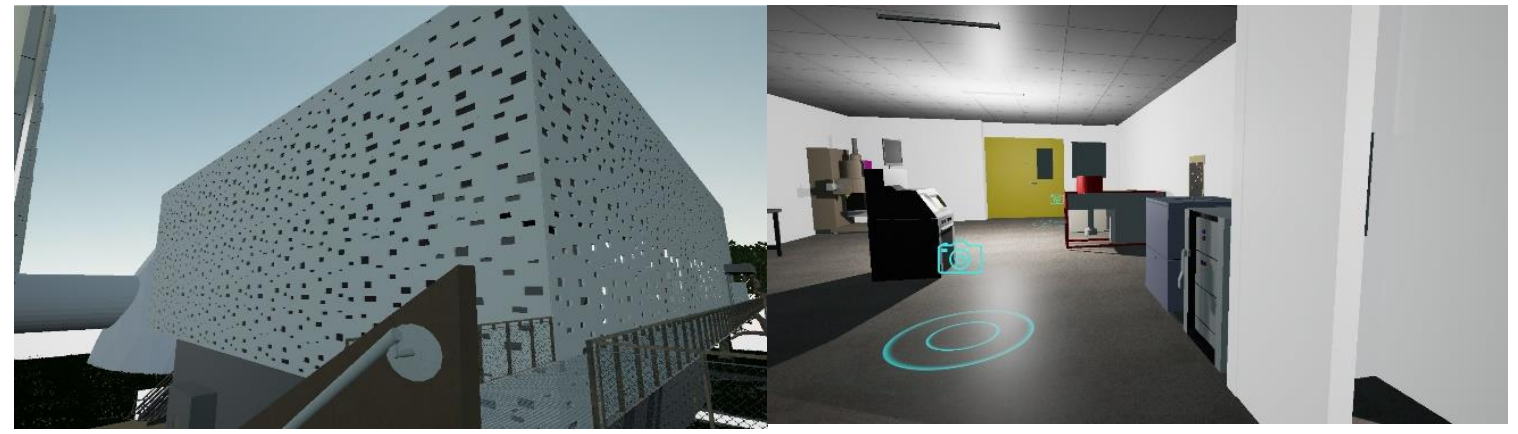

Fig.5. Bâtiment modélisé, extérieur (gauche), une salle (droite).

Un premier niveau d'interactivité a été implémenté : il est possible de se déplacer avec la souris et les manettes, mais il est aussi possible d'ouvrir et fermer des portes ainsi que prendre, déplacer, reposer quelques objets (microscopes, récipients etc.).

Pour plus de réalisme mais aussi pour pouvoir montrer des équipements qui n'ont pu être modélisés pendant le projet faute de temps, nous avons ajouté la possibilité d'afficher les images $360^{\circ}$ prises dans les salles blanches, en lieu et place des images de synthèse. Pour cela, l'utilisateur est informé qu'une photo à $360^{\circ}$ est disponible quand il voit deux cercles bleus concentriques au sol, surmontés d'un appareil photo (voir cercles sur la figure 5 , droite). Dans ce cas, quand l'utilisateur se place virtuellement sur ce cercle, il peut alors d'un simple clic remplacer l'image calculée (figure 6 gauche) par une vraie photographie 
panoramique comme ci-dessous (figure 6 droite) qu'il peut explorer en se tournant sur luimême.
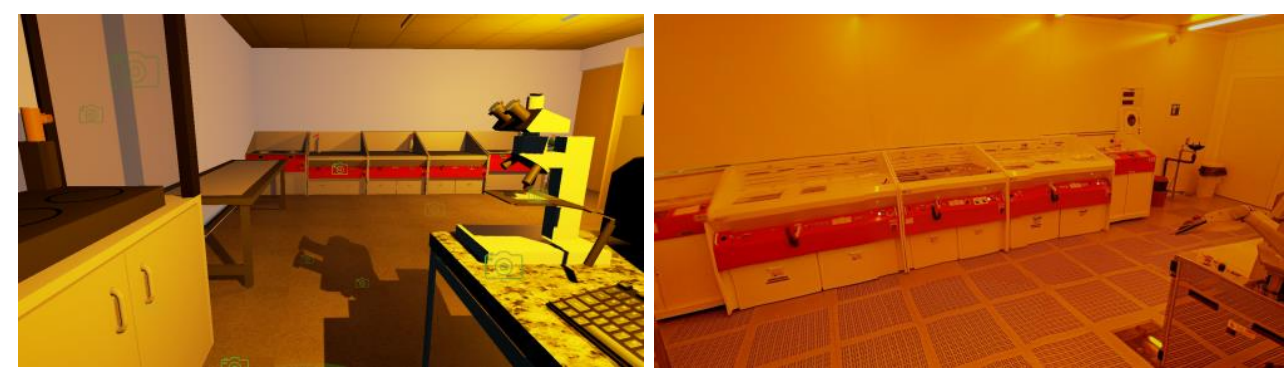

Fig.6. Version modélisée (gauche), photo $360^{\circ}$ (droite).

Signalons enfin que pour prendre en main le système, un tutoriel a été intégré au système. Il est exécuté lors du premier lancement de l'application.

\section{Conclusion}

Plusieurs points sont à améliorer à commencer par l'ergonomie et le ressenti pour la version casque immersif qui donne parfois le mal de mer. Nous souhaitons aussi poursuivre la modélisation de l'étage du dessus (arrivées d'air, filtres...) ainsi que du sous-sol dont seuls les murs sont visibles. Nous allons probablement refaire toutes les vues en format panoramique grâce au nouvel équipement que nous avons pu obtenir. L'interactivité est encore réduite, il faut étudier ce qui pourrait être fait notamment dans le cas d'utilisation de ce logiciel pour la formation plus avancée, par exemple pour simuler la manipulation des appareils. Nous envisageons aussi d'amener plus de vie dans cette univers virtuel, en commençant par l'ajout d'un avatar-guide qu'il suffirait de suivre comme un conférencier. Enfin, une version « en ligne » accessible depuis internet sur un simple PC ou tablette est à l'étude afin de permettre au plus grand nombre d'accéder à l'univers des salles blanches et de la micro technologie.

\section{Remerciements}

Nous remercions vivement Bruno Mercier qui a proposé et soutenu ce projet ainsi que le programme FINMINA (3) et le CNFM pour leur soutien.

\section{Références}

1. Philippe Fuchs, Guillaume Moreau, Alain Berthoz. «Traité de la réalité virtuelle volume 4: applications ». ISBN-10: 2911762479.

2. Cruz-Neira, Carolina; Sandin, Daniel J.; DeFanti, Thomas A.; Kenyon, Robert V.; Hart, John C. (1992-06-01). "The CAVE: audio visual experience automatic virtual environment". Communications of the ACM. 35 (6): 64-72. doi:10.1145/129888.129892. ISSN 0001-0782.

3. IDEFI-FINMINA : Initiative d'Excellence - Formation Innovante en MIcroélectronique et Nanotechnologies, ANR-11-IDFI-0017. Website: http://www.cnfm.fr/VersionFrancaise/actualites/ FINMINA.htm (Accès mars 2019)

4. GIP-CNFM: Groupement d'Intérêt Public - Coordination Nationale pour la formation en Microélectronique et en nanotechnologies. Website: http://wwww.cnfm.fr (Accès mars 2019) 